\title{
Patterning Multicolor Hybrid Perovskite Films via
}

\section{Top-Down Lithography}

Jonathon Harwell, James Burch, Alasdair Fikouras, Malte C Gather, Andrea Di Falco and Ifor David William Samuel*

SUPA, School of Physics and Astronomy, University of St Andrews, North Haugh, St Andrews, Fife, KY16 9SS. UK.

*Corresponding Author -idws@st-andrews.ac.uk

KEYWORDS: perovskite, light-emitting diode, lithography, patterning, photoluminescence, solution-processed, multicolor,

\section{ABSTRACT}

Lead halide perovskites have attracted great attention due to their excellent optoelectronic properties, with great progress being made in their performance as light emitting diodes (LEDs), photodiodes, and solar cells. Demonstrating large scale, high-resolution patterning of perovskites is a key enabling step to unlock their full potential for a range of optoelectronic applications. However, the development of a successful top-down lithography fabrication procedure has so far been hampered by the incompatibility of perovskite films with the solvents used during lithographic processes. Here, we perform a study on the effect of different lithographic solvents on perovskite films, and use this insight to develop a procedure for photolithography and electron beam lithography to pattern perovskite films. This procedure uses standard resists at low temperatures, and achieves micron scale features with flat tops. Furthermore we expand this platform to produce arrays of multicolor pixels for commercial perovskite LED display applications. 
Hybrid perovskites have proved to be a promising material for a range of optoelectronic applications including solar cells, ${ }^{1,}{ }^{2}$ light emitting diodes (LEDs), ${ }^{3-6}$ photodiodes, ${ }^{7,} 8$ light emitting field effect transistors, ${ }^{9,} 10$ and lasers. ${ }^{11,12}$ Hybrid perovskite devices combine excellent optoelectronic properties with solution processing. ${ }^{8,13}$ This enables the production of devices on low-cost and flexible plastic substrates, ${ }^{14}, 15$ with applications in deformable optoelectronic devices.

Applying these developments to commercial products such as multicolor displays or camera modules requires patterning perovskite films into arrays of individual devices, rather than bulk films. In industry, the preferred fabrication method for these devices is top-down lithography (TDL), in particular photolithography or electron beam lithography when nanoscale resolution is required. TDL produces high resolution patterning over large areas, whilst being cheap, fast, and highly reproducible. ${ }^{16,17}$

The general recipe for a lithography process has four key steps -1 ) a photoresist is spin-coated onto the material to be patterned, 2) the resist is then pre-baked and exposed to ultraviolet light (UV) or electron beams (e-beam), 3) the sample is placed in a developer solution (after optional post-exposure baking) to remove the unwanted resist, ready for step 4), where the resist pattern is transferred into the underlying sample by etching. Each of these steps presents harsh conditions which have the potential to cause damage to the material. This is particularly a problem for solution-processed semiconductors because they are easily dissolved, and are often relatively soft. Most varieties of hybrid perovskite are stable enough to withstand the processes in steps 2) and 4), but the polar and protic solvents involved in steps 1) and 3), typical of commercial lithography processes, are known to dissolve or degrade perovskite films. Hence TDL was widely believed to be incompatible with hybrid perovskites. ${ }^{16-19}$

In order to avoid the difficulties with lithographic solvents, researchers have developed a wide range of alternative methods to pattern perovskites without the use of TDL. Most notably, Kim et $a l^{19}$ and $\mathrm{Wu}$ et $a l^{17}$ exploited dewetting of the perovskite precursor to achieve single color features of high quality by patterning hydrophobic monolayers on the substrate, rather than patterning the perovskite directly, while several other groups have used nanoimprint patterning of the precursor solvents to force them to crystallize into the desired structure. ${ }^{20}, 21$ Nanoimprinting has also been used to thermally imprint gratings and photonic crystals directly 
onto a perovksite film. This method has even been shown to improve the photoluminescence properties of the films, resulting in lasers with improved threshold ${ }^{22}$. Fluorinated resists have also been employed by Lin et al., who used them in a lift-off process to achieve multicolor patterning on perovskite quantum dots, ${ }^{18}$ and Zakhidov et al., who patterned the perovskite by degrading it to $\mathrm{PbF}_{2}$ in specific areas rather than removing it completely. ${ }^{23}$ Additionally, Alias et al have shown that gas-assisted focussed ion beam etching is an effective method for achieving submicron patterning on the small scale. ${ }^{24}$ Finally, Song et al found that Poly(methyl methacrylate) (PMMA) can be safely deposited on perovskites without degrading them. ${ }^{25}$ PMMA is unsuitable as a photoresist due to its weak absorption in the near UV. ${ }^{26}$ But high quality features were achieved on perovskite microplatelets by using electron beam lithography, as long as the perovskite was not exposed to the developer solution (a propan-2-ol (IPA) and Methyl isobutyl ketone (MIBK) solution) for too long.

From an industrial perspective, the ideal patterning process would use commercial resists, which are widely available and have already been well optimised. In order to achieve large areas, the patterning process must also be compatible with UV lithography methods, and it must work at low baking temperatures in order to allow deposition on flexible substrates. To be compatible with creating electronic devices, the process also needs to create flat topped features. For this purpose, dry etch processes such as oxygen plasma or argon ion milling are preferable over wet etch or lift-off processing, due to their easier optimisation and high directionality. Finally, a process which could pattern multiple different materials onto the same substrate would enable more complex devices, such as directly emissive multicolor displays.

Despite the advances made in patterning perovskites, TDL remains the only option which could achieve all the goals described above, and hence it is important to find a way to make hybrid perovskites compatible with commercial resists. In this work, we demonstrate that high quality patterning of perovskite films via TDL is possible when using SU-8, a negative epoxy based resist sold by Microchem. While PMMA is ineffective as a photoresist in TDL, we found that it can act as an easily removable spacer layer to protect the perovskite film from the solvents used for the processing of SU-8. When the perovskite film is protected in this way, SU-8 can be deposited on the film and then patterned using standard TDL protocols at low temperatures. The normal photolithography process can then be followed, with oxygen plasma being used to etch 
the pattern into the PMMA and argon milling to etch the perovskite. This achieves perovskite features as small as $3 \mu \mathrm{m}$ across once the PMMA spacer is removed by washing in chloroform or toluene.

Our method is applicable to many varieties of perovskite, including 3D perovskites for photodiodes or layered perovskites for LEDs, and also works with electron beam lithography for features as small as $\sim 1 \mu \mathrm{m}$ (see figure S9). This modification to the industry standard method will greatly simplify the process of achieving high resolution patterning in perovskite films. This will enable further research into significant fields such as perovskite microlasers or photonic structures, whilst also removing a major barrier to the commercialisation of perovskite optoelectronic devices.

\section{RESULTS AND DISCUSSION}

\section{SINGLE COLOR PATTERNS}

To investigate the issues with lithographic solvents, we performed a study of the effects of the solvents involved in three major commercial resists on a methylammonium lead iodide $\left(\mathrm{CH}_{3} \mathrm{NH}_{3} \mathrm{PbI}_{3}\right)$ perovskite film. We first studied $\mathrm{SU}-8$, which at first appears to be a good candidate because hybrid perovskites are insoluble in the ethyl lactate (EC) developer used for step 3 (see figure S1). However, we found that spin-coating SU-8 photoresist directly onto any form of perovskite gives poor results, which is likely due to reaction with the cyclopentanone solvent or photoacids within the mixture (see figure S2). In the best case, the SU-8 forms a poor quality film which cannot be patterned by UV exposure (see figure S3), and in the worst case the perovskite is immediately degraded as shown in figure S4. This is in agreement with the findings of other groups. ${ }^{17,19,23}$

Other commercial resists run into similar problems. The $\mathrm{S} 1800$ series resist, a dye-based positive tone photoresist distributed by Microchem, appears to work well in steps 1 and 2, but fails in step 3, where the metal ion free developer solution (MF319) was found to instantly dissolve the perovskite film (see figure S1). Finally, PMMA is a basic electron beam resist, which forms good quality films when spin-coated on top of perovskites due to its solubility in orthogonal solvents such as anisole or chlorobenzene. However the IPA/water developer solution for PMMA was found to degrade the perovskite films (see e.g. figure S1). 
These results are problematic because it would seem that none of the available resists are suitable for all 3 steps, so we aimed to overcome this issue by combining two resists into one process. PMMA performs well in step 1, but poorly in 2 and 3, while SU-8 should perform well in steps 2 and 3 if only the issues in step 1 can be overcome. Therefore, our solution is to develop a process in which SU-8 and PMMA are used in combination to overcome the limitations of either material on its own. We use the PMMA as a spacer layer to protect the perovskite from the SU-8 solvent during spin-coating for step 1, while allowing the SU-8 film to be processed normally on top of the stack for steps 2 and 3. The resulting SU-8 pattern could then be etched into the perovskite film, with the only difference being the need to etch through the PMMA spacer first.

To test this approach, we first aimed to pattern a highly emissive perovskite film with potential applications for a perovskite LED (PeLED) display. This would demonstrate an immediate potential application of our patterning method, and would also allow us to easily observe any degradation caused by the processing by monitoring the photoluminescence quantum yield (PLQY) of the film. Layered perovskites have recently been used to produce highly efficient PeLEDs, $^{3-6}$ and so for this experiment we spin-coated $220 \mathrm{~nm}$ of $\mathrm{n}=3$ phenethylammonium(PEA)methylammonium(MA) lead bromide (PEA) $2(\mathrm{MA})_{2} \mathrm{~Pb}_{3} \mathrm{Br}_{10}$ onto a glass substrate (see Experimental section for details). The resulting films emitted at $\sim 515 \mathrm{~nm}$ (see figure S6) with a PLQY of roughly $55 \%$. The patterning protocol is illustrated in figure 1. Once the perovskite films were formed, $710 \mathrm{~nm}$ of PMMA (Microchem, A7 950) was spincoated onto the film, followed by $400 \mathrm{~nm}$ of SU-8 (Microchem, 2000.5). With the PMMA layer to protect the perovskite from the SU-8, it was found that the SU-8 would form films of similar thickness and quality to when it was coated on bare glass, and there was no visible degradation. This demonstrates that PMMA is effective at separating the SU-8 from the perovskite, and thus overcomes the problematic step 1 of the lithography process. 


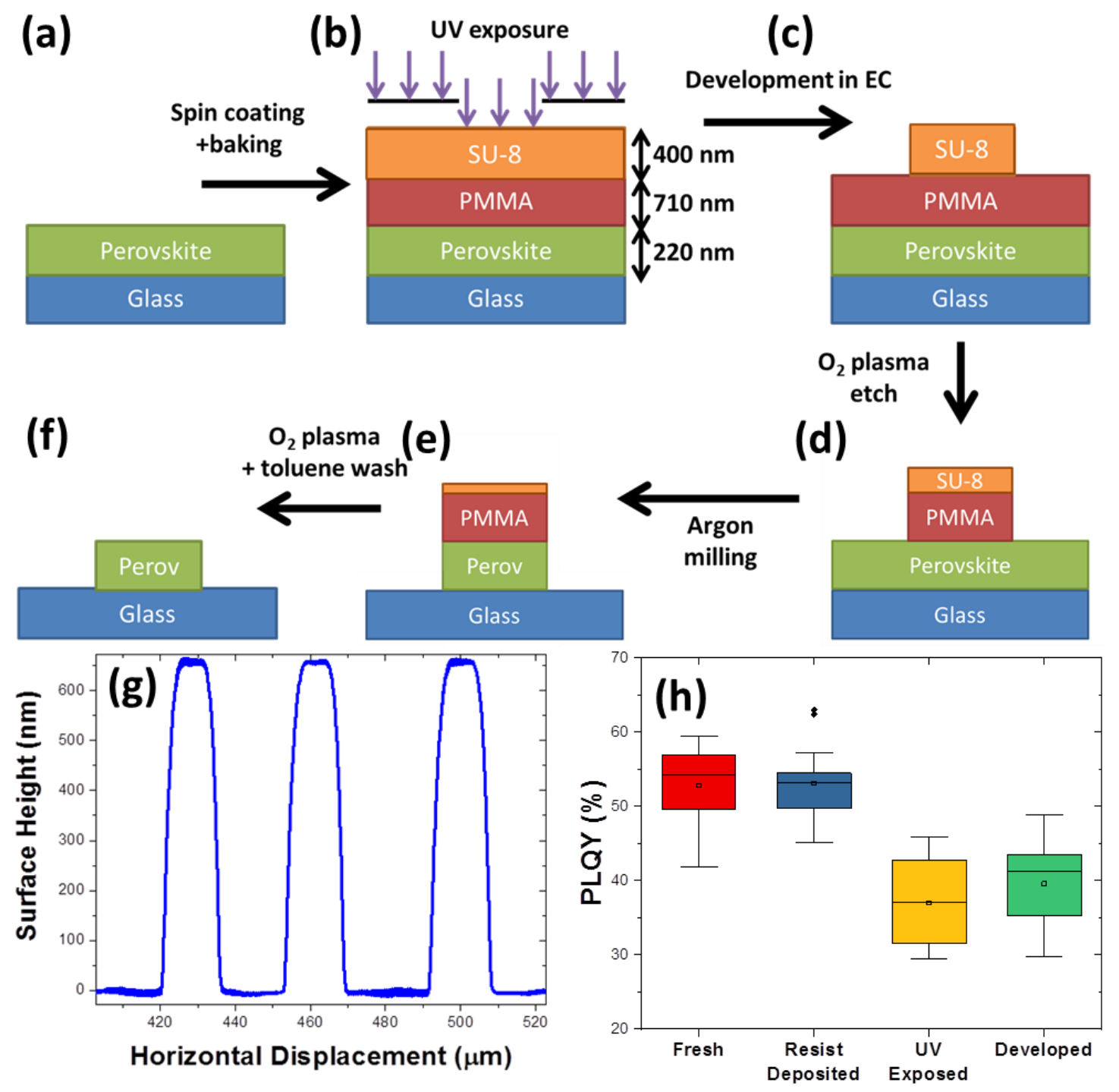

Figure 1 - The patterning process - (a) PMMA is spin coated onto the perovskite film, followed by SU-8 resist. (b) The resist is exposed via UV or electron beam, and then developed in EC. (c) The PMMA capping layer is etched away via oxygen plasma. (d) The perovskite is etched away via argon ion milling. (e) The remaining SU-8 is etched away with oxygen plasma, and the PMMA is washed away by immersion in chloroform or toluene, resulting in the final result (f). (g) The surface profile of the SU-8 features produced in (c). The pattern is stripes of $15 \mu \mathrm{m}$ width. (h) PLQY measurements of the $(\mathrm{PEA})_{2}(\mathrm{MA})_{2} \mathrm{~Pb}_{3} \mathrm{Br}_{10}$ film at different points in the lithography process. 
Once the SU-8 had been deposited and baked, the sample was exposed to UV light (figure 1(b)), using a shadow mask to transfer a pattern of $15 \mu \mathrm{m}$ stripes to the resist. The sample was then post baked and developed in EC (Microchem) for 60 seconds, resulting in the structure in figure 1(c). After development, the patterned features can be clearly seen on the sample, and surface profilometry shows that the SU-8 has indeed cleanly developed, resulting in clear features as shown in figure $1(\mathrm{~g})$. Note that the height of these features is larger than the initial thickness of the SU-8 layer because the EC developer acts as a slow wet etchant on the PMMA. This has no impact on the quality of features that can be achieved.

To ensure that the lithography process does not cause major degradation of the perovskite, we performed PLQY measurements on the perovskite film at every step of the processing. The PLQY was measured using $450 \mathrm{~nm}$ light in order to minimise any absorption in the SU-8 layer. Samples for this measurement were flood exposed in the UV stage so as to fairly measure the impact of the high intensity UV on the perovskite. As can be seen from the results on figure 1(h) the processing has an acceptably low impact on the PLQY. The PLQY remains unchanged by the resist deposition and baking process, but it is somewhat reduced by the exposure to UV - a known issue with this class of layered perovskite. ${ }^{27}$ The end result is that the PLQY drops from $55 \%$ to $40 \%$ over the entire process. This could conceivably be improved when using more photostable perovskite variants which contain caesium or formamidinium.

Once the patterns have been formed in the SU-8 (figure 1(c)), the remaining PMMA was etched away using oxygen plasma (figure 1(d)), and the pattern was etched into the hybrid perovskite layer using argon ion milling (figure 1(e)). Finally, any remaining SU-8 was etched away using oxygen plasma, and the remaining PMMA was then removed by immersing the sample in chloroform for $60 \mathrm{~s}$ (figure 1(f)). This final oxygen plasma step is necessary because we found that the SU-8 did not cleanly lift off when using solvent immersion alone, even after 10 minutes immersion with sonication. Figure 2 shows examples of the resulting patterns that can be achieved on perovskite films, with the photoresists being completely removed after the process. 

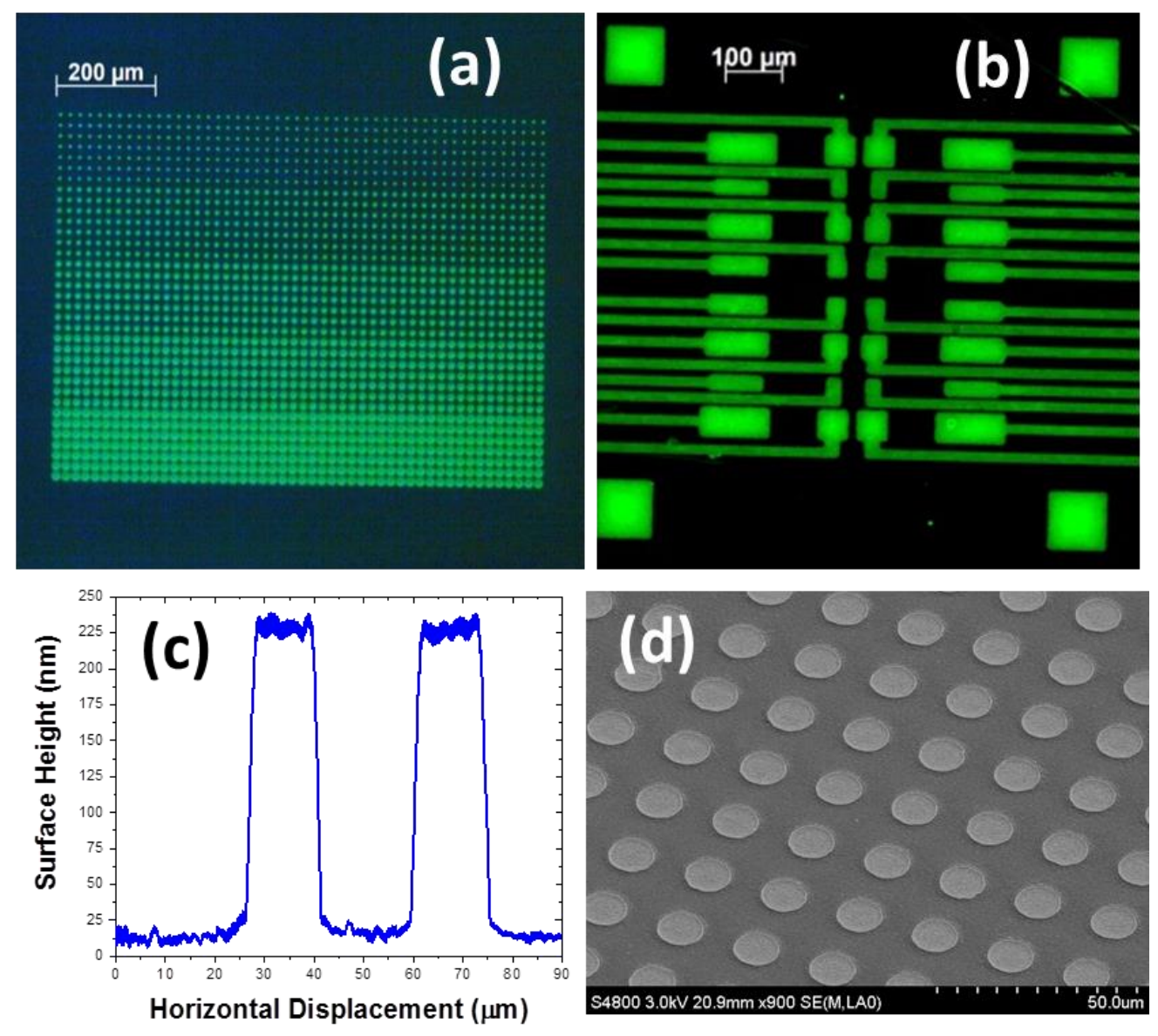

Figure 2 - $(\mathrm{PEA})_{2}(\mathrm{MA})_{2} \mathrm{~Pb}_{3} \mathrm{Br}_{10}$ perovskite films with the PMMA and SU-8 spacer layers removed post-patterning. (a) Microdisks of varying size between 3 and $17 \mu \mathrm{m}$. (b) An electrical device pattern with $15 \mu \mathrm{m}$ feature size. Microscope images are taken with a $5 \mathrm{x}$ objective in the dark field, with the samples illuminated with UV light. (c) Surface profilometry of the $15 \mu \mathrm{m}$ stripe features shown in part (b). The feature height of $220 \mathrm{~nm}$ indicates that all photoresist has been completely removed, leaving features which would work in an electrical device. (d) Angled scanning electron microscopy image of $\mathrm{CH}_{3} \mathrm{NH}_{3} \mathrm{PbI}_{3}$ microdisks patterned using this method.

Surface profilometry and angled scanning electron microscopy measurements show that the resulting features are sufficiently flat to be incorporated in monolithic electronic devices. To test the minimum feature size possible, arrays of microdisks of varying size were patterned, and the smallest microdisks that could be reliably patterned were $3 \mu \mathrm{m}$ in diameter (see figure S7 \& S8). To ensure that the patterned (PEA) $)_{2}(\mathrm{MA})_{2} \mathrm{~Pb}_{3} \mathrm{Br}_{10}$ films are sufficiently smooth to be used in electrical devices, the surface roughness of a patterned film was measured by surface 
profilometry (shown in figure S6). The root mean square roughness was found to be $1.6 \mathrm{~nm}$ over a $50 \mu \mathrm{m}$ scan and $4.5 \mathrm{~nm}$ over a $500 \mu \mathrm{m}$ scan. This closely matches the roughness reported in literature perovskite LED films, ${ }^{6}$ and indicates that the lithography process is not detrimental to the smoothness of a perovskite film.

An additional advantage of our fabrication platform is that it is compatible with electron beam lithography (EBL). EBL allows for the finest control over feature geometry, and is a common fabrication technique in both academia and industry. To show compatibility with EBL, we performed a standard EBL procedure on a perovskite/PMMA/SU-8 sample. After the EBL exposure, our samples were developed and etched with our standard procedure, resulting in feature sizes of $1.1 \mu \mathrm{m}$. The minimum feature size was limited by the minimum thickness of SU8 required to etch through the perovskite film, meaning that finer features could be achieved with

further optimisation on thinner or softer perovskite films. An additional consideration is that photolithography and EBL could be performed on the same sample before development, this means that large area and high resolution patterns can be combined, making our technique highly flexible.

\section{MULTICOLOR PATTERNS}

The above results will already have a multitude of uses for the fabrication of important devices such as microdisk lasers, photodiode arrays, or single color LED arrays. However a capability for multicolor arrays is important for fabricating high quality displays or color-sensitive cameras. The color of a perovskite film is mainly tuned by altering its composition, but it has recently been shown that gratings and photonic structures are also a highly effective way of tuning the film's apparent color both as an absorber $^{28}$ and as an emitter, ${ }^{29}$ allowing multiple colors to be seen on a single film. It is also possible to tune the color of a single film of perovskite nanowires ${ }^{30}$ or microplatelets ${ }^{31}$ using anion exchange techniques to selectively alter the bandgap the material. These methods could be combined with the lithography process described in this work to achieve multicolor patterns of perovskite nanoparticles on a large scale; however this method is less effective when working with bulk perovskite films because phase separation of the halides can result in unstable and poor quality photoluminescence. ${ }^{32}$ Hence a method for achieving a multicolor film from two separate solutions of completely different composition is an important challenge. 


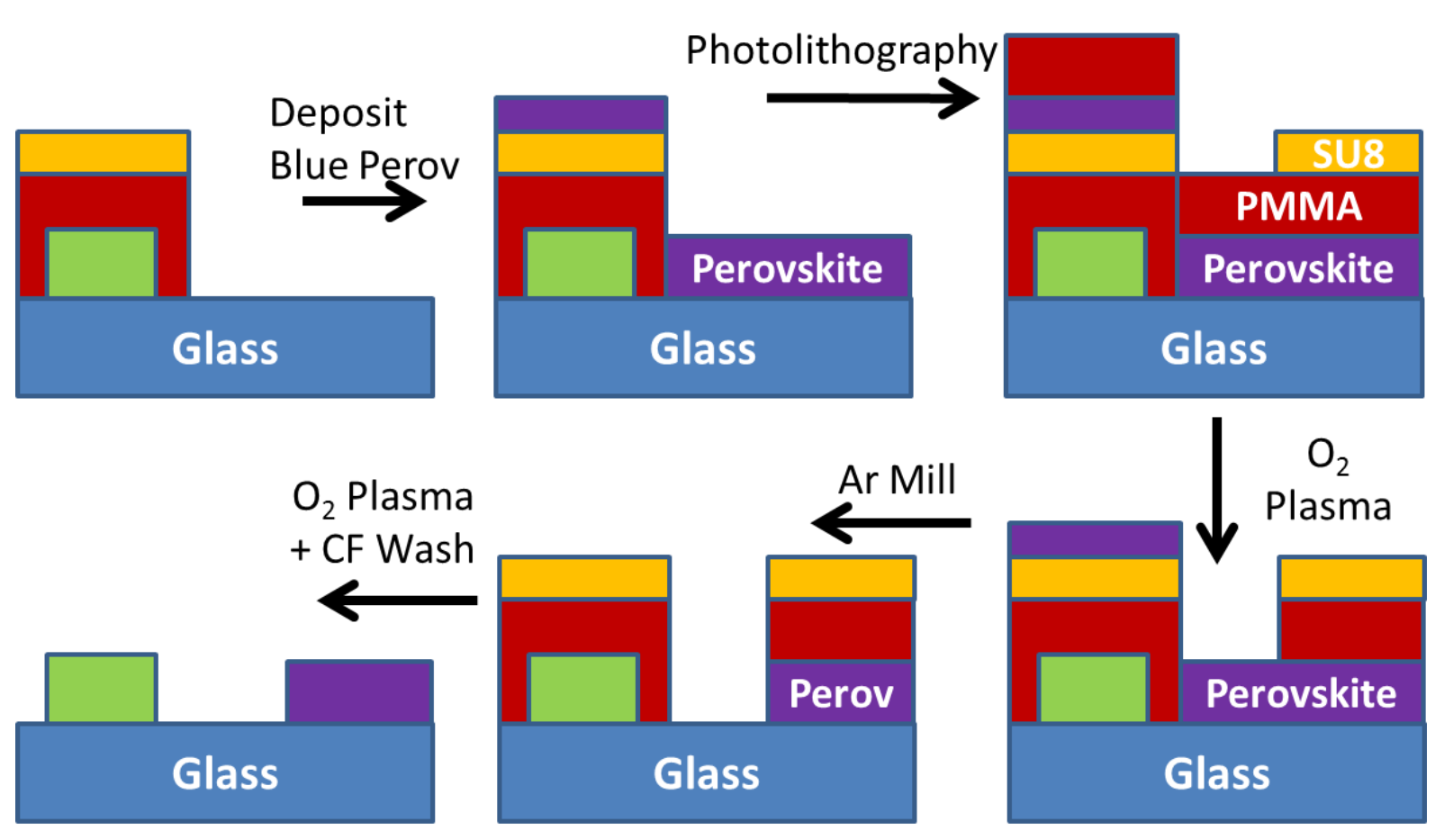

Figure 3 - The lithography process to achieve multicolor arrays. Note that the sidewalls of the original green perovskite film must be protected by PMMA.

Achieving multicolor perovskite films from two solutions is difficult because the solvent for the second color material will dissolve the material already on the substrate. Hybrid perovskites are particularly vulnerable to this issue because their films are much more soluble in their native solvent than most organic semiconductors, meaning they are instantly dissolved by contact with very small amounts of solvent. Therefore simply leaving the SU-8/PMMA capping layer on to protect the perovskite from the next solvent is not sufficient because the exposed sidewalls are rapidly dissolved by the second solution, creating an undercut of $\sim 100 \mu \mathrm{m}$ as shown in figure S10.

To overcome this issue, each pixel needs to be completely encapsulated by PMMA/SU-8 in order to prevent the pixels being dissolved. This can be achieved by spin-coating PMMA/SU-8 on the patterned sample, and then exposing an area slightly larger than the pixel in order to surround the pixel with PMMA/SU-8 on all sides as shown in figure S11. Once this is achieved, the photolithography process described in the previous section can be repeated in the way shown in figure 3 to achieve a multicolor pixel array. 


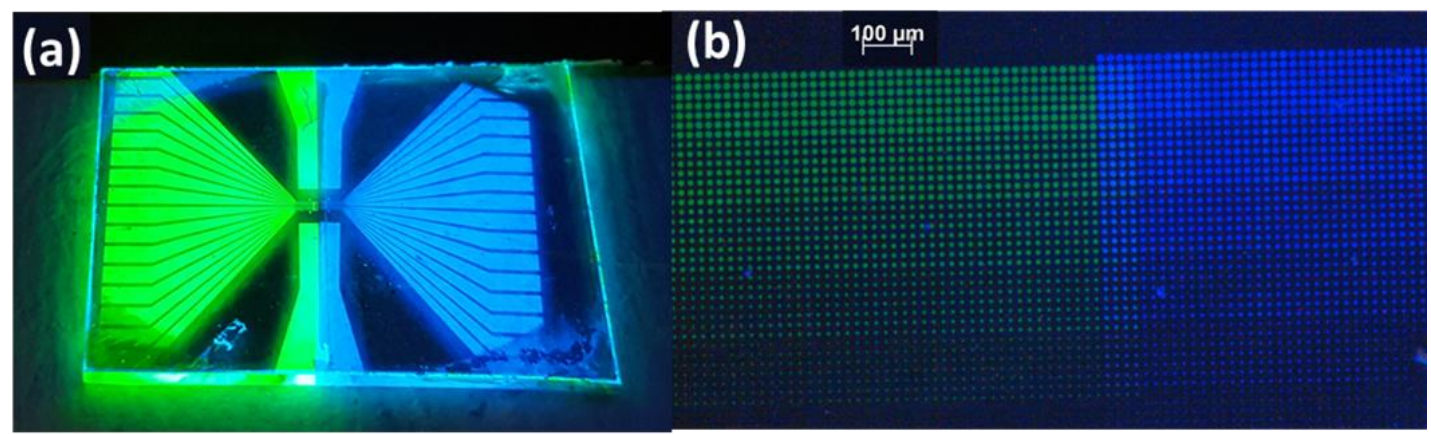

(c)

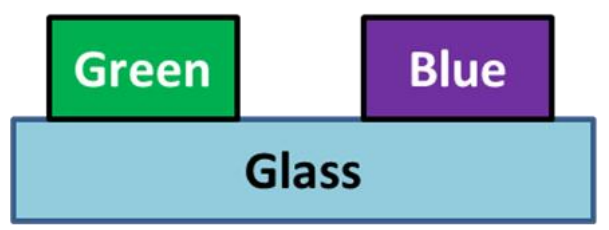

(d)

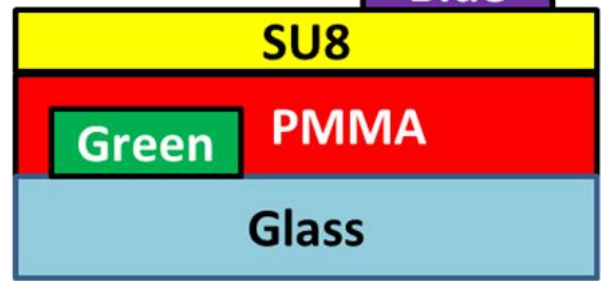

Figure 4 - (a) A two color perovskite pattern, fabricated by the method described above. The final cross section is shown in (c). (b) A two color array of microdisks made by the simplified method. This method requires less specialised masks, but results in the cross section shown in (d).

In figure 4 we show some simple demonstrations of two color samples using this method. Sample (a) is made by the method described above, using a chloride doped version of the same perovskite to produce the blue emission (see experimental for details). Sample (b) is made using a simplified method where the SU-8 is flood exposed after deposition, thus completely protecting the green pixels and allowing the SU-8 to be treated as a fresh substrate. This method results in a sample with the profile shown in figure 4 (d), where the pixels are slightly offset from each other. This simplified method will be useful in simpler devices such as color conversion layers where the slight parallax is not an issue. However the first method should be used for electrical devices where the pixels are required to share a common substrate.

\section{CONCLUSIONS}

In conclusion, we have clearly demonstrated TDL on hybrid perovskites. This method, previously thought to be incompatible with hybrid perovskites, can be performed using commercial resists with only minor modifications to the standard lithography procedures. By 
combining widely available resists with the use of a PMMA protection layer, excellent quality features are achieved whilst only causing minor degradation of the perovskite film. This method will make patterning of perovskite films much more accessible to the wider scientific community, enabling a wealth of potential applications for perovskites. With the use of a mask aligner or stepper, this method can also be repeated multiple times in order to produce arrays of multicolor pixels. With perovskite LEDs and photodiodes making such rapid progress in recent years, this capability to produce multicolor arrays could enable commercial production of large area perovskite displays or camera sensors.

\section{METHODS}

\section{MAKING THE PEROVSKITE FILMS}

For the green layered perovskite, an $\mathrm{n}=3$ stoichiometry precursor solution of $(\mathrm{PEA})_{2}(\mathrm{MA})_{2} \mathrm{~Pb}_{3} \mathrm{Br}_{10}$ was prepared by dissolving lead bromide $\left(\mathrm{PbBr}_{2}\right)$, phenethylammonium bromide (PEABr), and methylammonium bromide (MABr) in DMSO to make a $1 \mathrm{M}$ solution. Specifically, $367 \mathrm{mg}$ of $\mathrm{PbBr}_{2}, 75 \mathrm{mg} \mathrm{MABr}$, and $135 \mathrm{mg} \mathrm{PEABr}$ were dissolved in $1 \mathrm{ml}$ of DMSO by stirring at room temperature for 30 minutes. While the solution was dissolving, glass substrates were cleaned by sonication in acetone and then IPA for 5 minutes, followed by plasma ashing in oxygen plasma for 3 minutes. The solution was then spin-coated in an $\mathrm{N}_{2}$ filled glovebox $\left(\mathrm{O}_{2}\right.$ and water $\left.<0.1 \mathrm{ppm}\right)$ at $8000 \mathrm{RPM}$ for $60 \mathrm{~s}$. When the sample had been spinning for 15-20 seconds, an antisolvent made from chloroform containing $5 \mathrm{mgml}^{-1}$ 2,2',2"-(1,3,5Benzinetriyl)-tris(1-phenyl-1-H-benzimidazole) (TPBi) (Ossilla, unsublimed 99.8\%) was rapidly dropped onto the sample while spinning. The samples were then annealed at $90{ }^{\circ} \mathrm{C}$ for 10 minutes to complete conversion. It is important to keep the $\mathrm{O}_{2}$ count as low as possible, and to change the foil in the spin-coater after every $\sim 5$ coatings because contaminants in the local atmosphere can strongly impact the film quality.

The blue perovskite film was made using a chloride doped version of the same process. A high chloride solution was made by dissolving $278 \mathrm{mg} \mathrm{PbCl}_{2}, 135.4 \mathrm{mg} \mathrm{PEABr}$, and $45.22 \mathrm{mg}$ methylammonium chloride in $1 \mathrm{ml}$ DMSO. This solution was then mixed in 40:60 ratio with the pure bromide solution and then deposited via spin coating at 8000 RPM for 60 seconds. A 
solution of TPBi in chloroform was dripped on the sample after 10-15 s spinning, and the sample was annealed at $90{ }^{\circ} \mathrm{C}$ for 10 minutes.

To make films of $\mathrm{CH}_{3} \mathrm{NH}_{3} \mathrm{PbI}_{3}$, lead iodide and methylammonium iodide were dissolved in a 1:1 stoichiometry to a $1.4 \mathrm{M}$ concentration in a 9:1 DMF:DMSO solvent blend. The dissolved solution was then spin-coated at 4000 RPM for $30 \mathrm{~s}$ with an antisolvent of diethyl ether being dropped onto the substrate after 7 seconds of spinning. The resulting films were then annealed for 3 minutes at $100{ }^{\circ} \mathrm{C}$.

Once the films were fabricated, they were transferred out of the glovebox for photolithography. PMMA (A7 950 Microchem) was spin-coated onto the sample at 5000 RPM for $60 \mathrm{~s}$ and was then annealed at $90{ }^{\circ} \mathrm{C}$ for 5 minutes. Once the sample had cooled, a solution of SU-8 (2000.5, Microchem) was spin-coated onto the sample at 8000 RPM for $60 \mathrm{~s}$ before being annealed at 90 ${ }^{\circ} \mathrm{C}$ for 4 minutes. The film was then exposed to $365 \mathrm{~nm}$ UV light in a mask aligner for 2 minutes (Karl Suss MJB-3), with a post exposure bake of 2 minutes at $90{ }^{\circ} \mathrm{C}$. The pattern was developed in EC (microposit) for $60 \mathrm{~s}$ to produce the pattern. Samples exposed using e-beam were exposed with a standard electron beam lithography procedure with a RAITH eLine PLus lithography system. The electron energy was set to $30 \mathrm{keV}$, the aperture to $10 \mu \mathrm{m}$, and the dose $8.0 \mu \mathrm{Ccm}^{-2}$. They were then developed in the same way as the UV samples.

Post patterning, the remaining PMMA spacer was removed using low pressure oxygen plasma for 2 minutes. The etch rate was $200 \mathrm{~nm} /$ minute for PMMA and $100 \mathrm{~nm} /$ minute for SU-8. The samples were then transferred to a custom made chemically assisted ion beam etch (CAIBE) setup where they underwent argon ion milling at $500 \mathrm{ccm}, 1450$ volts for 10 minutes. The etch rate of the perovskite under these conditions was approximately $20 \mathrm{~nm} /$ minute, with the SU-8 etching at a similar rate. The remaining SU-8 capping layer was then removed by 4 minutes in oxygen plasma under the same conditions as before.

Surface profilometry measurements were taken using a Veeco Dektak 150 surface profilometer, and PLQY measurements were taken using a Hamamatsu U6039-05 setup. Scanning electron microscopy images were taken with a Hitachi S4800 SEM with an angle-controlled stage, and microscope images were taken in the dark field with a microscope, using a UV torch to excite the perovskite. 
ASSOCIATED CONTENT

SUPPORTING INFORMATION

The research data supporting this publication can be accessed at https://doi.org/10.17630/f06f61ab-b0ec-4aae-ba97-5ec49771a578

The following supporting information is available free of charge via the Internet at http://pubs.acs.org.

- Images of the impact of different solvents and photoresists on a CH3NH3PbI3 Film

- PL spectra and solution stoichiometry information on the (PEA)2(MA)2Pb3 Br10 films used in this study

- Scanning electron microscopy images of patterned microdisks

- Schematics detailing the difficulties with multicolor patterns, and the ways they were overcome

- A study on the impact of developing S1818 photoresist on a protected perovskite film ACKNOWLEDGEMENTS

This work was (partially) supported by the European Commission under the European Union's Horizon 2020 research and innovation programme, project MILEDI (grant agreement $\mathrm{n}^{\circ}$ 779373), and by the Engineering and Physical Sciences Research Council (EPSRC) of the UK Grants; EP/M025330/1, EP/L017008/1, and EP/M508214/1. We thank C. Keum and C. Murawski for the development of the photomasks used in figures 2(a) and 4(b).

\section{REFERENCES}

1. Chen, J. Z.; Park, N. G., Inorganic Hole Transporting Materials For Stable And High Efficiency Perovskite Solar Cells. J Phys Chem C 2018, 122, 14039-14063.

2. Wu, C. G.; Chiang, C. H.; Tseng, Z. L.; Nazeeruddin, M. K.; Hagfeldt, A.; Gratzel, M., High Efficiency Stable Inverted Perovskite Solar Cells Without Current Hysteresis. Energy Environ Sci 2015, 8, 2725-2733.

3. Chang, J.; Zhang, S. T.; Wang, N. N.; Sun, Y.; Wei, Y. Q.; Li, R. Z.; Yi, C.; Wang, J. P.; Huang, W., Enhanced Performance Of Red Perovskite Light-Emitting Diodes Through The 
Dimensional Tailoring Of Perovskite Multiple Quantum Wells. J Phys Chem Lett 2018, 9, 881886.

4. Yuan, M. J.; Quan, L. N.; Comin, R.; Walters, G.; Sabatini, R.; Voznyy, O.; Hoogland, S.; Zhao, Y. B.; Beauregard, E. M.; Kanjanaboos, P.; Lu, Z. H.; Kim, D. H.; Sargent, E. H., Perovskite Energy Funnels For Efficient Light-Emitting Diodes. Nat Nanotechnol 2016, 11, 872877.

5. Cho, H. C.; Jeong, S. H.; Park, M. H.; Kim, Y. H.; Wolf, C.; Lee, C. L.; Heo, J. H.; Sadhanala, A.; Myoung, N.; Yoo, S.; Im, S. H.; Friend, R. H.; Lee, T. W., Overcoming The Electroluminescence Efficiency Limitations Of Perovskite Light-Emitting Diodes. Science 2015, $350,1222-1225$.

6. Wang, N. N.; Cheng, L.; Ge, R.; Zhang, S. T.; Miao, Y. F.; Zou, W.; Yi, C.; Sun, Y.; Cao, Y.; Yang, R.; Wei, Y. Q.; Guo, Q.; Ke, Y.; Yu, M. T.; Jin, Y. Z.; Liu, Y.; Ding, Q. Q.; Di, D. W.; Yang, L.; Xing, G. C.; et al., Perovskite Light-Emitting Diodes Based On SolutionProcessed Self-Organized Multiple Quantum Wells. Nat Photonics 2016, 10, 699-704.

7. Kim, J. M.; Shin, D. H.; Choi, S. H., Highly-Flexible Perovskite Photodiodes Employing Doped Multilayer-Graphene Transparent Conductive Electrodes. Nanotechnology 2018, 29, 425203.

8. Lin, Q. Q.; Armin, A.; Lyons, D. M.; Burn, P. L.; Meredith, P., Low Noise, IR-Blind Organohalide Perovskite Photodiodes For Visible Light Detection And Imaging. Adv Mater 2015, 27, 2060-2064.

9. Maddalena, F.; Chin, X. Y.; Cortecchia, D.; Bruno, A.; Soci, C., AC-Driven Perovskite Light-Emitting Field-Effect Transistors. 2017 Conference On Lasers And Electro-Optics Europe \& European Quantum Electronics Conference (Cleo/Europe-Eqec) 2017.

10. Chin, X. Y.; Cortecchia, D.; Yin, J.; Bruno, A.; Soci, C., Lead Iodide Perovskite LightEmitting Field-Effect Transistor. Nat Commun 2015, 6, 7383.

11. Zhang, Q.; Su, R.; Du, W. N.; Liu, X. F.; Zhao, L. Y.; Ha, S. T.; Xiong, Q. H., Advances In Small Perovskite-Based Lasers. Small Methods 2017, 1,1700163 . 
12. Harwell, J. R.; Whitworth, G. L.; Turnbull, G. A.; Samuel, I. D. W., Green Perovskite Distributed Feedback Lasers. Sci Rep-Uk 2017, 7,11727.

13. Laboratory, N. R. E. NREL Solar Cell Efficiency Chart. Https://Www.Nrel.Gov/Pv/Assets/Pdfs/Pv-Efficiencies-07-17-2018.Pdf. (accessed Jan 2019)

14. Wu, Z. W.; Li, P.; Zhang, Y. K.; Zheng, Z. J., Flexible And Stretchable Perovskite Solar Cells: Device Design And Development Methods. Small Methods 2018, 2, 1800031.

15. Li, L. B.; Zhang, S. S.; Yang, Z. C.; Berthold, E. E. S.; Chen, W., Recent Advances Of Flexible Perovskite Solar Cells. J Energy Chem 2018, 27, 673-689.

16. Jeong, B.; Hwang, I.; Cho, S. H.; Kim, E. H.; Cha, S.; Lee, J.; Kang, H. S.; Cho, S. M.; Choi, H.; Park, C., Solvent-Assisted Gel Printing For Micropatterning Thin Organic-Inorganic Hybrid Perovskite Films. Acs Nano 2016, 10, 9026-9035.

17. Wu, J.; Chen, J. Y.; Zhang, Y. F.; Xu, Z. J.; Zhao, L. C.; Liu, T. H.; Luo, D. Y.; Yang, W. Q.; Chen, K.; Hu, Q.; Ye, F. J.; Wu, P.; Zhu, R.; Gong, Q. H., Pinhole-Free Hybrid Perovskite Film With Arbitrarily-Shaped Micro Patterns For Functional Optoelectronic Devices. Nano Lett 2017, 17, 3563-3569.

18. Lin, C. H.; Zeng, Q. J.; Lafalce, E.; Yu, S. T.; Smith, M. J.; Yoon, Y. J.; Chang, Y. J.; Jiang, Y.; Lin, Z. Q.; Vardeny, Z. V.; Tsukruk, V. V., Large-Area Lasing And Multicolor Perovskite Quantum Dot Patterns. Adv Opt Mater 2018, 6, 1800474.

19. Lee, W.; Lee, J.; Yun, H.; Kim, J.; Park, J.; Choi, C.; Kim, D. C.; Seo, H.; Lee, H.; Yu, J. W.; Lee, W. B.; Kim, D. H., High-Resolution Spin-On-Patterning Of Perovskite Thin Films For A Multiplexed Image Sensor Array. Adv Mater 2017, 29, 1702902.

20. He, X. X.; Liu, P.; Zhang, H. H.; Liao, Q.; Yao, J. N.; Fu, H. B., Patterning Multicolored Microdisk Laser Arrays Of Cesium Lead Halide Perovskite. Adv Mater 2017, 29, 1604510.

21. Yang, X. Y.; Wu, J.; Liu, T. H.; Zhu, R., Patterned Perovskites For Optoelectronic Applications. Small Methods 2018, 2, 1800110. 
22. Pourdavoud, N.; Wang, S.; Mayer, A.; Hu, T.; Chen, Y. W.; Marianovich, A.; Kowalsky, W.; Heiderhoff, R.; Scheer, H. C.; Riedl, T., Photonic Nanostructures Patterned By Thermal Nanoimprint Directly Into Organo- Metal Halide Perovskites. Adv Mater 2017, 29, 1605003.

23. Lyashenko, D.; Perez, A.; Zakhidov, A., High-Resolution Patterning Of Organohalide Lead Perovskite Pixels For Photodetectors Using Orthogonal Photolithography. Phys Status Solidi A 2017, 214, 1600302 .

24. Alias, M. S.; Yang, Y.; Ng, T. K.; Dursun, I.; Shi, D.; Saidaminov, M. I.; Priante, D.; Bakr, O. M.; Ooi, B. S., Enhanced Etching, Surface Damage Recovery, And Submicron Patterning Of Hybrid Perovskites Using A Chemically Gas-Assisted Focused-Ion Beam For Subwavelength Grating Photonic Applications. J Phys Chem Lett 2016, 7, 137-142.

25. Zhang, N.; Sun, W. Z.; Rodrigues, S. P.; Wang, K. Y.; Gu, Z. Y.; Wang, S.; Cai, W. S.; Xiao, S. M.; Song, Q. H., Highly Reproducible Organometallic Halide Perovskite Microdevices Based On Top-Down Lithography. Adv Mater 2017, 29, 1606205.

26. Johnstone, R. W.; Foulds, I. G.; Parameswaran, M., Deep-UV Exposure Of Poly(Methyl Methacrylate) At $254 \mathrm{~nm}$ Using Low-Pressure Mercury Vapor Lamps. J Vac Sci Technol B 2008, 26, 682-685.

27. Fang, H. H.; Yang, J.; Tao, S. X.; Adjokatse, S.; Kamminga, M. E.; Ye, J. T.; Blake, G. R.; Even, J.; Loi, M. A., Unravelling Light-Induced Degradation Of Layered Perovskite Crystals And Design Of Efficient Encapsulation For Improved Photostability. Adv Funct Mater 2018, 28, 1800305 .

28. Zhang, W.; Anaya, M.; Lozano, G.; Calvo, M. E.; Johnston, M. B.; Miguez, H.; Snaith, H. J., Highly Efficient Perovskite Solar Cells With Tunable Structural Color. Nano Lett 2015, $15,1698-1702$.

29. Gao, Y. S.; Huang, C.; Hao, C. L.; Sun, S.; Zhang, L.; Zhang, C.; Duan, Z. H.; Wang, K. Y.; Jin, Z. W.; Zhang, N.; Kildishev, A. V.; Qiu, C. W.; Song, Q. H.; Xiao, S. M., Lead Halide Perovskite Nanostructures For Dynamic Color Display. Acs Nano 2018, 12, 8847-8854. 
30. Dou, L.; Lai, M. L.; Kley, C. S.; Yang, Y. M.; Bischak, C. G.; Zhang, D. D.; Eaton, S. W.; Ginsberg, N. S.; Yang, P. D., Spatially Resolved Multicolor CsPbX3 Nanowire Heterojunctions Via Anion Exchange. P Natl Acad Sci USA 2017, 114, 7216-7221.

31. Zhang, N.; Wang, K. Y.; Wei, H. H.; Gu, Z. Y.; Sun, W. Z.; Li, J. K.; Xiao, S. M.; Song, Q. H., Postsynthetic And Selective Control Of Lead Halide Perovskite Microlasers. J Phys Chem Lett 2016, 7, 3886-3891.

32. Hoke, E. T.; Slotcavage, D. J.; Dohner, E. R.; Bowring, A. R.; Karunadasa, H. I.; Mcgehee, M. D., Reversible Photo-Induced Trap Formation In Mixed-Halide Hybrid Perovskites For Photovoltaics. Chem Sci 2015, 6, 613-617. 